\section{Recognizing facial emotion}

SIR-The amygdala has been implicated in emotion and emotional memory in humans and experimental animals ${ }^{1,2}$. Some of us $^{3}$ have reported impaired recognition of emotion in facial expressions (especially fear) in a 30-year-old woman (S.M.) with a nearly complete bilateral lesion of the amygdala due to congenital UrbachWiethe disease. This suggested that the amygdala may be essential not only for emotional learning, but also for recognition of emotion in facial expressions. Here we report data from two men who survived herpes simplex encephalitis (E.P. ${ }^{4}$

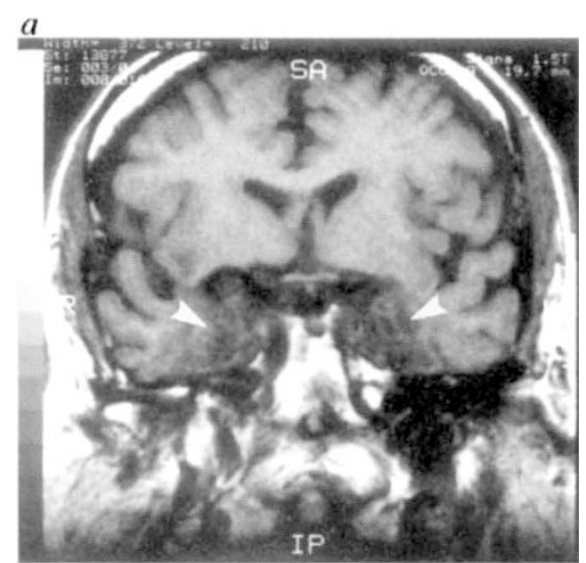

b

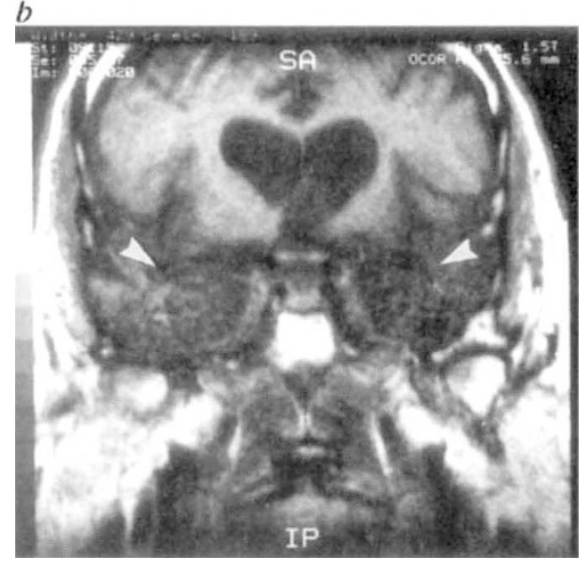

FIG. 1 a, Coronal T1-weighted magnetic res onance image of patient E.P. showing extensive bilateral damage to the amygdaloid complex (arrows). The damage extends rostrally to the temporal pole and caudally to include the hippocampal formation. There is also severe atrophy of the entorhinal, perirhinal and parahippocampal cortices. b, Coronal T1-weighted magnetic resonance image of patient G.T. at a similar rostrocaudal level, showing severe bilateral temporal lobe damage (arrows). The damage extends through the anterior $7.0 \mathrm{~cm}$ of G.T.'s left temporal lobe and through the anterior $5.0 \mathrm{~cm}$ of his right temporal lobe. The lesion bilaterally includes the amygdaloid complex, hippocampus, entorhinal, perirhinal and parahippocampal cortices as well as the inferior, middle and superior temporal gyri: There is also bilateral damage in the insular cortex, medial and orbital frontal cortex, and cingulate gyrus. and G.T., aged 73 and 59 years, respectively), with complete bilateral lesions of the amygdala and additional temporal lobe structures (Fig. 1). Despite this, they appear unimpaired in recognizing facial expressions of emotion, including fear. E.P and G.T. are profoundly amnesic but have IQ scores in the normal range.

E.P. and G.T. were tested twice, once using the exact materials and procedure reported by Adolphs et al. ${ }^{3}$ (Fig. 2), and again using a slightly modified version of the same experiment. E.P. and G.T. rated normally the same facial expressions that S.M. rated abnormally. For the modified test, in which the emotional adjectives varied randomly from trial to trial, the two patients performed similarly to four ageand education-matched controls in every respect (correlations between groups $>0.70$ for each of the six emotions). For faces consistent with the emotion being rated, the patients obtained an average rating of 4.6 (4.8 for fear) on a 0-5 scale; control rating was 4.3 (4.3 for fear).

There are two possibilities consistent with these data. One possibility is that bilateral damage to the amygdala together with damage outside the amygdala is required to impair the recognition of facial emotion. Intracranial lesions resulting from Urbach-Wiethe disease can extend beyond the amygdala to include other structures ${ }^{5.6}$. In addition to her amygdala lesion, S.M. has minimal damage to the entorhinal cortex ${ }^{2}$ on the left side. Moreover, postmortem histopathological analysis may reveal damage not detected by current neuroimaging techniques. Damage to the basal ganglia is reported to impair the recognition of facial emotion ${ }^{7}$, although it is unclear that such impairment is similar to that of S.M.

A more likely possibility is that amygdala lesions impair the recognition of emotion in facial expressions only if these lesions occur early in development, rather than in adulthood. The Urbach-Wiethe disease that led to S.M.'s amygdaloid lesions is congenital, whereas E.P. and G.T. sustained their lesions after the age of 50 years. D.R., a 51-year-old woman who is also deficient in recognizing facial emotion $^{8}$, sustained partial bilateral lesions to the amygdala and to the right basal ganglia in adulthood. However, D.R. has a history of epilepsy and a broader deficit than S.M. in processing emotions. Perhaps a combination of factors, including a congenital lesion, low full-scale IQ $(\mathrm{S} . \mathrm{M} .=86$, D.R. $=87$ ) and/or additional brain damage, could determine how readily other strategies are available for recognizing facial emotion.

The present report suggests that in the

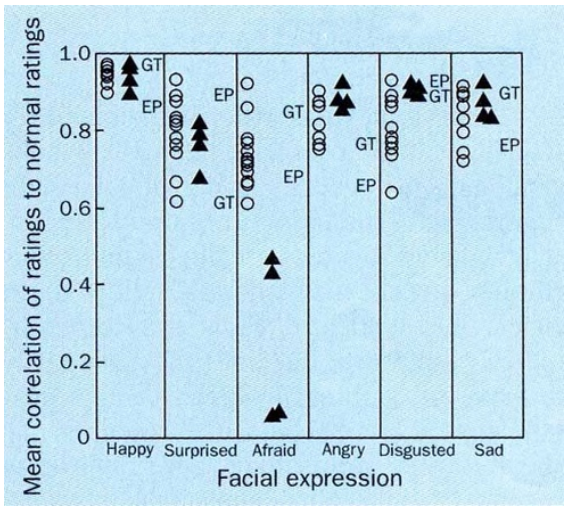

FIG. 2 Correlations of ratings of facial expressions on emotional adjectives with ratings given by previously studied ${ }^{3}$ normal controls $(n=7)$. Mean Pearson correlations are shown for the ratings given by brain-damaged controls (circles; $n=12$ ), S.M.'s ratings (triangles show results for 4 experiments), E.P.'s ratings (EP; 1 expt), and G.T.'s ratings (GT; 1 expt). Data for the braindamaged controls and S.M. are from Adolphs et al. ${ }^{3}$. Data for E.P. and G.T. are from an experiment that replicated the procedures used therein. Recognition of facial expression of human emotion was tested by presenting on a screen and one at a time 6 different expressions of facial emotion (each displayed by 6 different faces). Subjects rated each face with respect to 9 adjectives (the 6 emotions shown in the figure, as well as awake, sleepy or interested).

absence of damage early in life, recognition of facial emotion in adults does not have an absolute dependence on the amygdala. These data and conclusions are fully compatible with an important role for the amygdala in the experience and expression of emotion. Tasks that assess the recognition of fear in facial expressions may not require the actual experience of fear.

Stephan B. Hamann, Lisa Stefanacci

Department of Psychiatry,

University of California,

San Diego, California 92093, USA

\section{Larry R. Squire}

Departments of Psychiatry and Neurosciences,

University of California,

San Diego, California 92093, USA and

VA Medical Center,

San Diego, California 92161, USA

Ralph Adolphs, Daniel Tranel

Hanna Damasio, Antonio Damasio

Department of Neurology,

University of lowa, College of Medicine,

lowa City, lowa 52242, USA

1. LeDoux, J. Behav. Brain Res. 58, 69-79 (1993).

2. Bechara, A. et al Science 269, 1115-1118 (1995).

3. Adolphs, R., Tranel, D., Damasio, H. \& Damasio, A. Nature 372, 669-672 (1994).

4. Squire, L. \& Knowiton, B. Proc. natn. Acad. Sci. U.S.A. 92, 12470-12474 (1995).

5. Friedman, D., Mathews, R. \& Swanepoel, P. S. Afr. med. . 65, 734-735 (1984)

6. Newton, F., Rosenberg, R., Lampert, P. \& O'Brien, J. Neurology 21, 1205-1213 (1971).

7. Jacobs, D., Shuren, J., Bowers, D. \& Heilman, K Neurology 45, 1696-1702 (1995).

8. Young, A., Hellawell, D., van de Wal, C. \& Johnson, M. Neuropsychologia (in the press) 